\title{
The association between the transfer of emergency department admitted patients to inpatient hallways and outcomes of oncology patients
}

\author{
Charles Lim, Matthew C. Cheung, Maureen E. Trudeau, Kevin R. Imrie, Ben de Mendonca, Simron Singh * \\ Sunnybrook Health Sciences Centre, Odette Cancer Centre, Toronto, ON, Canada
}

Received: October 27, 2014

Accepted: January 15, 2015 Online Published: January 26, 2015

DOI: $10.5430 /$ jha.v4n2p1

URL: http://dx.doi.org/10.5430/jha.v4n2p1

\begin{abstract}
Objective: A protocol was implemented to ease Emergency Department (ED) crowding by moving suitable admitted patients into inpatient hallway beds (HALL) or off-service beds (OFF) when beds on an admitting service's designated ward (ON) were not available. This study assessed the impact of hallway and off-service oncology admissions on ED patient flow, quality of care and patient satisfaction.

Methods: Retrospective and prospective data were collected on patients admitted to the medical oncology service from Jan 1 to Dec 31, 2011. Data on clinician assessments and time performance measures were collected. Satisfaction surveys were prospectively administered to all patients.

Results: Two hundred and ninty-seven patients (117 HALL, 90 OFF, 90 ON) were included in this study. There were no significant differences between groups for frequency of physician assessments, physical exam maneuvers at initial physician visit, time to complete vital signs or time to medication administration. The median (IQR) time spent admitted in the ED prior to departure from the ED was significantly longer for HALL patients (5.53 hrs [1.59-13.03 hrs]) compared to OFF patients (2.00 hrs [0.37-3.69 hrs]) and ON patients $(2.18 \mathrm{hrs}[0.15-5.57 \mathrm{hrs}])(p<.01)$. Similarly, the median (IQR) total ED length of stay was significantly longer for HALL patients (13.82 hrs [7.43-20.72 hrs]) compared to OFF patients (7.18 hrs [5.72-11.42 hrs]) and ON patients $(9.34 \mathrm{hrs}[5.43-14.06 \mathrm{hrs}])(p<.01)$. HALL patients gave significantly lower overall satisfaction scores with mean $(S D)$ satisfaction scores for HALL, OFF and ON patients being $3.58(1.20), 4.23(0.58)$ and $4.29(0.69)$ respectively $(p$ $<.01)$. Among HALL patients, $58 \%$ were not comfortable being transferred into the hallway and $4 \%$ discharged themselves against medical advice.

Conclusions: The protocol for transferring ED admitted patients to inpatient hallway beds did not reduce ED length of stay for oncology patients. The timeliness and frequency of clinical assessments were not compromised; however, patient satisfaction was decreased.
\end{abstract}

Key Words: Emergency department, Crowding, Patient flow, Oncology, Inpatient hallway, Quality of care

\section{Introduction}

\subsection{Background}

Emergency department (ED) crowding is one of the most important issues facing the delivery of efficient and high- quality medical care in North America ${ }^{[1]}$ The underlying causes of ED crowding are complex and multifactorial, which include increasing patient complexity, decreasing hospital bed resources, and insufficient support staff resources. ${ }^{[2]}$ However, studies have repeatedly found board-

\footnotetext{
* Correspondence: Simron Singh; Email: Simron.Singh@sunnybrook.ca; Address: Sunnybrook Health Sciences Center, Odette Cancer Center Room T2-039, 2075 Bayview Avenue, Toronto, Canada.
} 
ing of admitted patients within the ED awaiting transfer to a ward bed to be the most important contributor to ED crowding. ${ }^{[3,4]}$ The consequences of ED crowding include delays in antibiotic administration ${ }^{[5-7]}$ poorer pain management, ${ }^{[8]}$ increased adverse events ${ }^{[9]}$ and increased patient mortality. ${ }^{[10]}$ Patient satisfaction also declines as ED crowding and ED patient boarding become increasingly problematic. ${ }^{[11]}$ The subset of oncology inpatients is not immune from the effects of a crowded ED. Cancer patients well known to a tertiary care center may be diverted to another hospital site without cancer expertise due to ED crowding. ${ }^{[12]}$

The Canadian Association of Emergency Physicians (CAEP) issued a position statement proposing that all admitted patients be transferred out of the ED within two hours of the decision to admit. ${ }^{[13]}$ The Ontario Ministry of Health and Long Term Care also issued a province-wide mandate for admitted patients to remain in the ED for no longer than 24 hours. To meet these targets, CAEP has recommended implementing full capacity protocols that would involve transferring admitted patients from the ED to inpatient hallways. Viccellio et al. retroactively reviewed this approach at their institution. ${ }^{[14]}$ With carefully selected patients chosen for temporary inpatient hallway placement, the authors did not find any increase in mortality or need for intensive care unit transfer for hallway bed admissions compared to standard bed admissions. However, the scope of this study was limited as it did not collect data on patient satisfaction, clinician assessments or time to interventions. Other studies have also found greater patient preference for inpatient hallway boarding and greater satisfaction compared to the option of remaining in the ED. ${ }^{[15,16]}$

\subsection{Importance}

There have not been any studies addressing hallway transfers specifically in an oncology patient population. Oncology patients are a unique subgroup of medical inpatients as their care typically involves a highly specialized interdisciplinary team of clinicians and nurses. The complex nature of their disease and complications of chemotherapy or radiation treatment are also distinguishing factors. ${ }^{[2]}$ Lastly, there are often psychosocial and symptom management issues for oncology patients, particularly those with advanced disease or those receiving end-of-life care.

Our institution implemented a full capacity protocol in October 2009 to ease ED crowding by moving suitable admitted patients from the ED into inpatient hallway beds. A similar policy was implemented in which patients were admitted to available beds in other areas of the hospital (offservice beds), if a bed designated for the admitting medical service (on-service bed) was not available.

\subsection{Goals}

This study assessed the impact of hallway and off-service admissions on patient flow in the ED, clinician assessments and patient satisfaction for cancer patients admitted to the medical oncology service. We hypothesized that the transfer of ED admitted patients to inpatient hallways would improve ED patient flow. Furthermore, we hypothesized that the unique complex care needs of oncology patients would make them unsuitable for hallway admission and that their clinical care and patient satisfaction would be adversely affected.

\section{Methods}

\subsection{Study design}

We prospectively identified a cohort of patients admitted to the medical oncology service from the ED between January 1 and December 31, 2011. All patients admitted to inpatient hallway beds under the medical oncology service during this time period were considered. The hospital full capacity protocol specified that patients could only be admitted to hallway beds if they did not have requirements for infection control isolation, telemetry, suctioning of secretions, or excessive supplementary oxygen. For each patient admitted to a hallway bed, one patient admitted to an on-service bed and one patient admitted to an off-service bed was randomly selected for review. Computerized bed assignment reports were reviewed daily, and on each instance where an inpatient hallway admission occurred, a randomly chosen on-service and off-service patient with the same date of admission was selected for review. On days where patients were admitted to a hallway bed but there were no corresponding admissions to on-service or off-service beds, only patients in hallway beds were included. This yielded comparison groups of 90 patients admitted to on-service and offservice beds respectively. Patients admitted to the radiation oncology or surgical oncology services were not included. Patients were excluded if they were transferred from outside hospitals, admitted from outpatient clinics, admitted on an elective basis, or transferred from the ICU to an inpatient unit. We approached all study participants to administer patient satisfaction surveys and we subsequently collected retrospective data from chart reviews.

\subsection{Outcomes}

Our primary clinical outcomes were time-in-motion measures of ED flow, including time from triage to admission and ED length of stay. These were derived from electronically captured time stamps including time of ED triage, time of admission decision, and time of ED departure. Secondary outcomes included patient-reported satisfaction markers including overall satisfaction with care during their admission as well as attitudes towards hallway admission and care. Other secondary outcomes included timeliness and frequency of clinician assessments. 


\subsection{Study ethics}

The study was approved by our institution's research ethics board. Financial support for this study was provided through the Academic Health Science Center Alternative Funding Plan Innovation Fund, funded by the Ontario Ministry of Health and Long Term Care.

\subsection{Study setting and population}

Sunnybrook Health Sciences Center (SHSC) is a universityaffiliated teaching hospital located in Toronto, Ontario with an annual ED census of 54,070 in 2011. The Odette Cancer Center (OCC) located on the SHSC campus is a highly specialized comprehensive cancer center serving southern Ontario and is the 6th largest cancer center in North America. OCC oncology patients in need of emergent inpatient care are most often admitted to an oncology ward in SHSC via the ED. In 2011, 1,013 patients were admitted to the inpatient medical oncology service.

SHSC implemented a full capacity protocol involving the transfer of admitted patients boarding in the ED to inpatient hallway beds that became effective on October 20, 2009. Suitable patients had no requirement for isolation, telemetry, suctioning of secretions, or excessive supplementary oxygen. Each inpatient unit accommodated up to two patients in hallway beds. When the appropriate trigger was met, the hospital bed flow coordinator was notified and hallway beds were assigned to admitted patients boarding in the ED. Another policy facilitating the admission of patients to beds other than those located on the admitting service's designated ward (off-service beds) was also in place. Patients could be admitted to off-service beds regardless of whether the full capacity protocol was activated or not.

\subsection{Data collection}

Retrospective data was collected from multiple sources including inpatient charts, the electronic patient record (Oasis) and the emergency department information system (iSoft). A trained research associate abstracted data for all included patients. A second research associate reviewed a random sample of 20 charts to ensure data validity. Demographic information was collected including gender and current treatment regimens. We collected clinical data including assessments by nurses and physicians, medication administration and diagnostic investigations. We examined time-in-motion performance markers including time from triage to admission decision, time from admission decision to departure from ED, and time spent in hallway beds. Lastly, we collected data on hospital bed occupancy rates from computerized bed management administrative databases.

A research associate approached all included patients within 24 hours of their arrival at the designated inpatient unit and asked them to complete a survey instrument that assessed their comfort with their hallway/standard bed admission, their perceived safety in care, and their attitudes towards wait times. The research associate then administered surveys after obtaining informed consent recording either written responses or verbal responses. All patients were asked about their overall satisfaction during their admission using a 5-point Likert scale with 5 defined as completely satisfied, 3 defined as neutral and 1 defined as completely unsatisfied.

\subsection{Data analysis}

Data analysis was performed using GraphPad Prism software (La Jolla, California, USA). Descriptive analysis was performed to determine the effect of the policy on ED time measures, time to key interventions, and patient satisfaction. Continuous data were reported as means with standard deviations $(S D)$ or medians with interquartile range (IQR) and binary data were reported as proportions. Comparisons between the hallway and standard bed groups were done using ANOVA and Student's $t$-test for continuous variables or $\chi^{2}$ test for categorical variables.

\section{Results}

We identified 117 patients admitted to an inpatient hallway bed under the oncology service and included them in our analysis. In our analysis of ED patient flow, there were no significant differences in the median time from ED triage to admission decision (see Table 1). The median (IQR) time spent admitted in the ED (i.e. from admission decision to ED departure) was significantly longer for HALL patients (5.53 hrs [1.59-13.03 hrs]) compared to OFF patients (2.00 hrs [0.37-3.69 hrs]) and ON patients (2.18 hrs [0.15-5.57 hrs]) $(p=.0004)$. Similarly, the median (IQR) total ED length of stay from triage to ED departure was significantly longer for HALL patients (13.82 hrs [7.43-20.72 hrs]) compared to OFF patients (7.18 hrs [5.72-11.42 hrs]) and ON patients (9.34 hrs [5.43-14.06 hrs]) $(p=.0006)$.

Upon arrival to the designated ward, median (IQR) time to first complete vital signs and median (IQR) time to first medication administration for HALL patients was $0.37 \mathrm{hrs}$ (0.06-1.21 hrs) and 6.02 hrs (1.63-14.88 hrs) respectively. There were not any significant differences between groups for these clinical time performance measures. The median (IQR) time spent in the hallway for HALL patients was $5.72 \mathrm{hrs}$ (2.20-14.05 hrs). Of 117 HALL patients, $12(10 \%)$ were in the hallway for less than one hour, $71(61 \%)$ were in the hallway between 1-12 hours, and 34 (29\%) were in the hallway for more than 12 hours. Five (4\%) HALL patients voluntary discharged themselves against medical advice, whereas no OFF or ON patients had voluntary discharges.

During the study period, total hospital occupancy at SHSC exceeded $100 \%$ for 180 days (49\%). For inpatient beds allocated to the medical oncology service, occupancy exceeded $100 \%$ for 312 days (85\%) and exceeded $110 \%$ for 273 days 
(75\%). Baseline characteristics for patients admitted to in- the first 48 hours and $43 \%$ had a documented chest or abpatient hallways (HALL), off-service non-oncology wards dominal exam at the first physician visit after arrival to the (OFF) and the on-service oncology ward (ON) were similar inpatient ward. There were no significant differences be(see Table 2). The site of primary malignancy for patients tween groups for either frequency of physician clinical asin each group is also described in Table 2. HALL patients sessments in the first 48 hours or physical exam maneuvers were seen by physicians an average of $6.3( \pm 3.3)$ times in at initial physician assessment.

Table 1: Key time performance measures for patients admitted to inpatient hallways, off-service beds and on-service beds

\begin{tabular}{|c|c|c|c|c|}
\hline & Hallway $(n=117)$ & Off-service $(n=90)$ & On-service $(\mathbf{n}=90)$ & $P$ value \\
\hline \multicolumn{5}{|l|}{ ED Length of Stay (hrs) } \\
\hline $\begin{array}{l}\text { Time from triage to decision to admit (median, } \\
\text { IQR) }\end{array}$ & $6.15(3.17-8.97)$ & $5.30(3.05-7.33)$ & $5.56(3.31-9.70)$ & .56 \\
\hline $\begin{array}{l}\text { Time from decision to admit to departure from ED } \\
\text { (median, IQR) }\end{array}$ & $5.53(1.59-13.03)$ & $2.00(0.37-3.69)$ & $2.18(0.15-5.57)$ & .0004 \\
\hline Total ED length of stay (median, IQR) & $13.82(7.43-20.72)$ & $7.18(5.72-11.42)$ & $9.34(5.43-14.06)$ & .0006 \\
\hline \multicolumn{5}{|l|}{ Time to Clinical Interventions (hrs) } \\
\hline Time to first complete vital signs (median, IQR) & $0.37(0.06-1.21)$ & $0.30(0-2.06)$ & $0.29(0-2.45)$ & .078 \\
\hline Time to medication administration (median, IQR) & $6.02(1.63-14.88)$ & $6.04(2.19-13.88)$ & $6.57(2.16-15.46)$ & .85 \\
\hline Total Time in Hallway (hrs) (median, IQR) & $5.72(2.20-14.05)$ & & & \\
\hline Time in Hallway $<1 \mathrm{hr}$ & $12(10 \%)$ & & & \\
\hline Time in Hallway 1-12 hrs & $71(61 \%)$ & & & \\
\hline Time in Hallway $12-24$ hrs & $27(23 \%)$ & & & \\
\hline Time in Hallway $>24$ hrs & $7(6 \%)$ & & & \\
\hline
\end{tabular}

Table 2: Patient characteristics and clinical assessments for patients admitted to inpatient hallways, off-service beds and on-service beds

\begin{tabular}{lllll}
\hline & Hallway (n = 117) & Off-service (n = 90) & On-service (n = 90) & P value \\
\hline Gender & & & & \\
Female & $51(44 \%)$ & $47(52 \%)$ & $43(48 \%)$ & .47 \\
Male & $66(56 \%)$ & $43(48 \%)$ & $47(52 \%)$ & \\
On Active Chemotherapy & & & & \\
Yes & $27(23 \%)$ & $14(16 \%)$ & $26(29 \%)$ & .10 \\
No & $91(77 \%)$ & $76(84 \%)$ & $64(71 \%)$ & \\
Site of Primary Malignancy & & & & \\
Breast & $17(15 \%)$ & $14(16 \%)$ & $10(11 \%)$ & \\
Central Nervous System & $5(4 \%)$ & $1(1 \%)$ & $2(2 \%)$ & \\
Dermatologic & $2(2 \%)$ & $3(3 \%)$ & $5(6 \%)$ & \\
Gastrointestinal & $28(24 \%)$ & $20(22 \%)$ & $10(11 \%)$ & \\
Genitourinary & $10(8 \%)$ & $7(8 \%)$ & $2(2 \%)$ & \\
Head and Neck & $4(3 \%)$ & $1(1 \%)$ & $15(17 \%)$ & \\
Hematologic & $23(20 \%)$ & $28(31 \%)$ & $14(15 \%)$ & \\
Lung & $16(14 \%)$ & $7(8 \%)$ & $4(4 \%)$ & \\
Prostate & $10(8 \%)$ & $4(4 \%)$ & $5(6 \%)$ & \\
Unknown & $2(2 \%)$ & $5(6 \%)$ & $5.78(2.57)$ & .10 \\
Clinical Assessments & & & $30(33 \%)$ & .22 \\
MD visits within first 48 hours (mean, SD) & $6.28(3.27)$ & $5.48(1.83)$ & $29(32 \%)$ & \\
Chest or Abdomen exam at first MD visit & $50(43 \%)$ & &
\end{tabular}


We attained a $37 \%$ response rate (113 of 297) for patient satisfaction surveys. Patients in all three groups mostly agreed that wait times were acceptable and that every effort was made to make them comfortable (see Table 3 ). There were significant differences in the proportion of patients that were satisfied with their overall care between the HALL (67\%), OFF $(92 \%)$ and ON (87\%) patient groups. HALL patients gave significantly lower overall satisfaction scores with the mean $(S D)$ satisfaction scores for HALL, OFF and ON patients being $3.58(1.20), 4.23(0.58)$ and $4.29(0.69)$ respec- tively ( $p=.0008$ ). Of the 36 HALL patient respondents, 26 $(72 \%)$ were told why they were placed in the hallway and $10(28 \%)$ were given the option to transfer to a hallway bed. Twenty-one (58\%) HALL patients felt uncomfortable being transferred into the hallway and $13(33 \%)$ did not feel that they were receiving appropriate care in the hallway. Hallway patients indicated in qualitative responses that their primary concerns were lack of privacy, too much noise and light, lack of communication devices, and increased risk of infection transmission.

Table 3: Satisfaction survey results for patients admitted to inpatient hallways, off-service beds and on-service beds

\begin{tabular}{|c|c|c|c|c|}
\hline & Hallway $(n=36)$ & Off-service $(n=39)$ & On-service $(n=38)$ & $P$ value \\
\hline Wait times were acceptable and reasonable for treatment & $26(72 \%)$ & $30(77 \%)$ & $28(74 \%)$ & \\
\hline Every effort was made to make the patient comfortable & $28(78 \%)$ & $31(79 \%)$ & $28(74 \%)$ & \\
\hline \multicolumn{5}{|l|}{ Satisfaction with care under admission to Oncology } \\
\hline Satisfied to some degree & $24(67 \%)$ & $36(92 \%)$ & $33(87 \%)$ & \\
\hline Score (mean, $S D$ ) & $3.58(1.20)$ & $4.23(0.58)$ & $4.29(0.69)$ & .0008 \\
\hline
\end{tabular}

\section{Discussion}

There are many anticipated benefits to rapid patient transfer out of the ED to inpatient hallways including improved ED flow and moving patients geographically closer to inpatient physicians and nursing staff. ${ }^{[15]}$ In addition, an inpatient hallway transfer protocol makes overflow a hospital-wide problem as opposed to an ED-only problem, therefore shifting more accountability to inpatient units and giving them greater incentive to improve patient flow. ${ }^{[17]}$ However, the potential flow benefits of such a policy need to be weighed against the impact on patient safety and patient satisfaction. Oncology patients in particular may be more adversely affected by hallway admission due to the complex nature of their disease, associated psychosocial issues, and unique dependence on highly specialized interdisciplinary skill sets to address their care needs. To our knowledge, this study is the first to examine the impact of hallway admission specifically for an oncology patient population.

We did not find any negative impact on physician clinical assessments for HALL patients compared to patients admitted to standard ward beds. There were no significant delays in having complete vital signs done or administering medications for HALL patients. These findings are consistent with previous studies that found no association between hallway boarding and medication delays or adverse events. ${ }^{[14,18]}$ We acknowledge that the eligibility criteria for inpatient hallway admission may introduce some selection bias. However, these criteria were developed and implemented institution-wide as temporary hallway beds did not have infrastructure capabilities to support isolation, telemetry or high flow oxygen. Though we did not report on mortality in our prospectively evaluated patient population, transfers to inpatient hallways did not appear to compromise direct patient care.

Implementing the full capacity protocol at SHSC did not reduce the median ED length of stay for oncology patients. Instead, HALL patients spent significantly more time admitted in the ED prior to departure from the ED compared to patients admitted to standard ward beds. There was no significant difference in ED length of stay for patients admitted to off-service or on-service beds. This finding was not congruent with several other studies that have found reduced ED length of stay with moving admitted patients out of the ED. ${ }^{[19-21]}$

Many contributing factors may explain this discrepancy. Firstly, the increased ED length of stay may have been more closely linked to overall hospital occupancy rates rather than ED volumes and crowding. Overall hospital occupancy exceeded $100 \%$ for nearly half of the study period. Though we were unable to correlate day-to-day hospital occupancy with ED length of stay, we hypothesize that ED length of stay for hallway patients would be most affected when hospital occupancy exceeded its surge capacity. In this scenario, hallway transfers may be ineffective if all potential hallway beds are occupied, leaving no further physical space for transferring admitted patients awaiting inpatient beds in the ED. Therefore, the increased ED length of stay may have reflected the underlying conditions triggering activation of the hallway transfer protocol, rather than the impact of the protocol itself. Secondly, the scope of this study was focused on a cohort of oncology patients and did not capture data for patients admitted to other hospital services. Our study focused specifically on medical oncology patients, and there was only a single inpatient unit where hallway beds could be assigned for these patients. In contrast, other studies examined broader patient populations where patients could be 
assigned to multiple inpatient units. Thirdly, unlike other investigators that had extensive experience with inpatient hallway transfers at their institutions, ${ }^{[14,21,22]}$ the full capacity protocol at SHSC was implemented only one year prior to the study period and was not yet a mature corporate procedure.

Despite increases in ED boarding time for HALL patients, the ED length of stay for oncology patients at SHSC was not significantly greater compared to studies at other institutions. A 2010 study by Wong et al. at another Toronto hospital reported median ED length of stay between 5 to 8 hours for oncology inpatients and between 12 to 16 hours for general internal medicine inpatients. ${ }^{[24]}$ Garson et al. reported mean ED length of stay between 8 to 12 hours among all patients boarding in the ED. ${ }^{[15]}$ In our study, $10 \%$ of patients admitted to hallway beds spent less than 1 hour in the hallway. Singer et al. and Viccellio et al. reported that the proportions of hallway patients that were moved to an inpatient ward bed in less than one hour were $53 \%$ and $50 \%$ respectively. ${ }^{[22,23]}$ This may have reflected the greater experience with the hallway transfers protocol at their institution or greater inpatient bed capacity.

Multiple studies have shown patient preference for inpatient hallway boarding over ED boarding. ${ }^{[16,25]}$ However, these surveys were conducted in ED patients prior to their actual admission. Our study focused on satisfaction and perceived quality of care for patients that actually experienced hallway admission compared to patients admitted to standard ward beds. We found that oncology patients were generally unhappy with their care in the hallway as shown by the increase in voluntary discharges and the significant decrease in overall satisfaction scores. More than half of patients assigned to hallway beds were uncomfortable with being transferred to the hallway, and only $67 \%$ of hallway patients agreed they were receiving appropriate care in the hallway.

The satisfaction survey results indicated that implementing the full capacity protocol at our institution had a negative impact on patient satisfaction. This finding contrasted previous studies in a broader patient population where patients preferred inpatient hallway boarding with no change in satisfaction scores. ${ }^{[15]}$ Unique characteristics specific to the oncology patient population may explain this difference. Firstly, active or recent treatment with immunosuppressing chemotherapy may heighten the sensitivity of oncology patients towards enforcing adequate infection control measures relative to other patients. In addition, oncology patients may experience more frequent and prolonged hospital admissions due to complications from their disease. This may also predispose to greater hesitancy with hallway admission as opposed to more familiar ward bed admission. These findings support our hypothesis that oncology patients are poor candidates for hallway admission due to their unique care requirements and complex disease.
Our findings demonstrate a need to refine the existing full capacity protocol and implement alternative strategies to improve patient flow at our institution. Reducing overall hospital occupancy rates remains a key corporate priority. A CAEP position statement recommends that governments invest in additional hospital infrastructure and acute care beds to achieve target hospital occupancy rates of $85 \% .^{[26]}$ In addition, coordinating early discharge of inpatients before noon has been extremely effective in reducing ED boarding in simulation models ${ }^{[27]}$ and is recommended as a high impact solution to ED crowding by the American College of Emergency Physicians Boarding Task Force. ${ }^{[28]}$ Our institution is actively incentivizing early discharge planning across all inpatient wards.

Several limitations apply to our study. Our study was conducted at a single academic institution with a designated inpatient medical oncology service, thus limiting the generalizability of the data. As with any retrospective chart review, incomplete chart documentation result in missing data is an inherent weakness. The research associate abstracting data was aware of the study protocol and hypothesis. However, main time measures including admission, transfer and medication administration times were taken from administrative data to reduce bias. Our prospective sample size was not large enough to comment on clinical outcomes such as mortality or medical complications. However, clinician assessment performance markers did not indicate any compromise in care received by any of the study groups. There may have been selection bias between the HALL, ON and OFF patient groups, due to the eligibility criteria for hallway admission outlined in the full capacity protocol. The survey data is limited by possible response bias as $37 \%$ of patients completed the survey. Some patients could not be approached for surveys if they were admitted after hours when a research associate was not available. The administration of the survey by a research associate during the hospitalization may have also introduced bias. Patient participants may have been reluctant to reveal their true opinions for fear of compromising their care in hospital, even though the survey instructions explicitly stated that their answers would not impact their clinical care. ${ }^{[15,25]}$ Lastly, our study did not address staff satisfaction with the full capacity protocol. Buy-in from health care providers is a key element to successfully implementing a full capacity protocol, and further studies are needed to investigate hospital and staff factors influencing strategies to address overcrowding.

\section{Conclusions}

Implementing a full capacity protocol involving transferring ED admitted patients to inpatient hallway beds at our institution did not meet its objective of reducing ED length of stay for oncology patients. We found that for oncology patients, hallway admission did not compromise direct clinical care but decreased patient satisfaction. It is unclear if this policy 
is beneficial to patient care or to relieving hospital crowding pressures. While full capacity protocols have been successfully implemented at other institutions, our study demonstrates a need for further research into patient, staff and hospital factors that influence the success of such strategies. Further refinements to the existing full capacity protocol and alternative solutions such as early discharge timing and increasing acute care bed capacity need to be considered.

\section{Disclosure}

Study previously presented at: 2013 ASCO Quality Care Symposium, San Diego, CA, USA.

\section{Acknowledgements}

We would like to acknowledge Dr. Steven Shadowitz, Darren Hefferon and Florina Weisenberg for their assistance.

\section{References}

[1] J.M. Pines, J.A. Hilton, E.J. Weber, A.J. Alkemade, H. Al Shabanah, P.D. Anderson, et al. International perspectives on emergency department crowding. Academic Emergency Medicine: Official Journal of the Society for Academic Emergency Medicine. 2011; 18: 1358-1370. PMid: 22168200. http://dx.doi.org/10.1111/j $.1553-2712.2011 .01235 . \mathrm{x}$

[2] Derlet, R.W., J.R. Richards. Overcrowding in the nation's emergency departments: complex causes and disturbing effects. Annals of Emergency Medicine. 2000; 35: 63-68. http://dx.doi .org/1 0.1016/S0196-0644(00)70105-3

[3] Pines, J.M., M.L. McCarthy. Executive summary: interventions to improve quality in the crowded emergency department. Academic Emergency Medicine : Official Journal of the Society for Academic Emergency Medicine. 2011; 18: 1229-1233. PMid: 22168183. http://dx.doi.org/10.1111/j.1553-2712.2011.01228.x

[4] McCarthy, M.L., S.L. Zeger, R. Ding, S.R. Levin, J.S. Desmond, J. Lee, et al. Crowding delays treatment and lengthens emergency department length of stay, even among high-acuity patients. Annals of Emergency Medicine. 2009; 54: 492-503.e4.

[5] Fee, C., E.J. Weber, C.A. Maak, P. Bacchetti. Effect of emergency department crowding on time to antibiotics in patients admitted with community-acquired pneumonia. Annals of Emergency Medicine. 2007; 50: 501-9, 509.e1.

[6] Pines, J.M., A.R. Localio, J.E. Hollander, W.G. Baxt, H. Lee, C. Phillips, et al. The impact of emergency department crowding measures on time to antibiotics for patients with community-acquired pneumonia. Annals of Emergency Medicine. 2007; 50: 510-516. PMid: 17913298. http://dx.doi.org/10.1016/j.annemergm ed.2007.07.021

[7] Pines, J.M., J.E. Hollander, A.R. Localio, J.P. Metlay. The association between emergency department crowding and hospital performance on antibiotic timing for pneumonia and percutaneous intervention for myocardial infarction. Academic Emergency Medicine : Official Journal of the Society for Academic Emergency Medicine. 2006; 13: 873-878. PMid: 16766743. http://dx.doi.org/10. 1111/j.1553-2712.2006.tb01741.x

[8] Hwang, U., L.D. Richardson, T.O. Sonuyi, R.S. Morrison. The effect of emergency department crowding on the management of pain in older adults with hip fracture. Journal of the American Geriatrics Society. 2006; 54: 270-275. PMid: 16460378. http://dx.doi.o $\mathrm{rg} / 10.1111 / \mathrm{j} .1532-5415.2005 .00587 . \mathrm{x}$

[9] Pines, J.M., C.V. Pollack Jr, D.B. Diercks, A.M. Chang, F.S. Shofer, J.E. Hollander. The association between emergency department crowding and adverse cardiovascular outcomes in patients with chest pain. Academic Emergency Medicine : Official Journal of the Society for Academic Emergency Medicine. 2009; 16: 617-625. PMid: 19549010. http://dx.doi.org/10.1111/j.1553-271 $2.2009 .00456 . x$

[10] Sprivulis, P.C., J.A. Da Silva, I.G. Jacobs, A.R. Frazer, G.A. Jelinek. The association between hospital overcrowding and mortality among patients admitted via Western Australian emergency de- partments. The Medical Journal of Australia. 2006; 184: 208-212. PMid: 16515429

[11] Bernstein, S.L., D. Aronsky, R. Duseja, S. Epstein, D. Handel, U. Hwang, et al. The effect of emergency department crowding on clinically oriented outcomes. Academic Emergency Medicine: Official Journal of the Society for Academic Emergency Medicine. 2009; 16: 1-10. PMid: 19007346. http://dx.doi.org/10.1111/j.1 553-2712.2008.00295.x

[12] Schull, M.J., L.J. Morrison, M. Vermeulen, D.A. Redelmeier. Emergency department overcrowding and ambulance transport delays for patients with chest pain. CMAJ: Canadian Medical Association Journal = Journal De L'Association Medicale Canadienne. 2003; 168: 277-283.

[13] Bullard, M.J., B. Unger, J. Spence, E. Grafstein, CTAS National Working Group. Revisions to the Canadian Emergency Department Triage and Acuity Scale (CTAS) adult guidelines. Cjem. 2008; 10: 136-151. PMid: 18371252.

[14] Viccellio, A., C. Santora, A.J. Singer, H.C. Thode Jr, M.C. Henry. The association between transfer of emergency department boarders to inpatient hallways and mortality: a 4-year experience. Annals of Emergency Medicine. 2009; 54: 487-491. PMid: 19345442. http: //dx.doi.org/10.1016/j.annemergmed.2009.03.005

[15] Garson, C., J.E. Hollander, K.V. Rhodes, F.S. Shofer, W.G. Baxt, J.M. Pines. Emergency department patient preferences for boarding locations when hospitals are at full capacity. Annals of Emergency Medicine. 2008; 51: 9-12, 12. e1-3.

[16] Richards, J.R., G. Ozery, M. Notash, P.E. Sokolove, R.W. Derlet, E.A. Panacek. Patients prefer boarding in inpatient hallways: correlation with the national emergency department overcrowding score. Emergency Medicine International. 2011; 840459. PMid: 22235374.

[17] Pines, J.M., D.M. Yealy. Advancing the science of emergency department crowding: measurement and solutions. Annals of Emergency Medicine. 2009; 54: 511-513. PMid: 19573948. http: //dx.doi.org/10.1016/j.annemergmed.2009.05.023

[18] Liu, S.W., Y. Chang, J.S. Weissman, R.T. Griffey, J. Thomas, S. Nergui, et al. An empirical assessment of boarding and quality of care: delays in care among chest pain, pneumonia, and cellulitis patients. Academic Emergency Medicine : Official Journal of the Society for Academic Emergency Medicine. 2011; 18: 1339-1348. PMid: 21692902. http://dx.doi.org/10.1111/j.1553-271 2.2011.01082. $\mathrm{x}$

[19] Liew, D., D. Liew, M.P. Kennedy. Emergency department length of stay independently predicts excess inpatient length of stay. The Medical Journal of Australia. 2003; 179: 524-526. PMid: 14609414.

[20] Richardson, D.B. The access-block effect: relationship between delay to reaching an inpatient bed and inpatient length of stay. The Medical Journal of Australia. 2002; 177: 492-495. PMid: 12405891.

[21] Khare, R.K., E.S. Powell, G. Reinhardt, M. Lucenti. Adding more beds to the emergency department or reducing admitted patient boarding times: which has a more significant influence on emergency department congestion? Annals of Emergency Medicine. 
2009; 53: 575-585. PMid: 18783852. http://dx.doi.org/10. 1016/j . annemergmed. 2008.07.009

[22] Viccellio, P., J.A. Zito, V. Sayage, J. Chohan, G. Garra, C. Santora, et al. Patients overwhelmingly prefer inpatient boarding to emergency department boarding. The Journal of Emergency Medicine. 2013; 45: 942-946. PMid: 24063879. http://dx.doi.org/10. $1016 / j \cdot$ jemermed.2013.07.018

[23] Singer, A.J., H.C. Thode Jr, P. Viccellio, J.M. Pines. The association between length of emergency department boarding and mortality. Academic Emergency Medicine: Official Journal of the Society for Academic Emergency Medicine. 2011; 18: 1324-1329. PMid: 22168198. http://dx.doi.org/10.1111/j.1553-2712.2011 $.01236 . \mathrm{x}$

[24] Wong, H.J., D. Morra, M. Caesar, M.W. Carter, H. Abrams. Understanding hospital and emergency department congestion: an examination of inpatient admission trends and bed resources. Cjem. 2010; 12: 18-26. PMid: 20078914
[25] Walsh, P., V. Cortez, H. Bhakta. Patients would prefer ward to emergency department boarding while awaiting an inpatient bed. The Journal of Emergency Medicine. 2008; 34: 221-226. PMid: 17976825. http://dx.doi.org/10.1016/j.jemermed. 2007.05 .012

[26] Canadian Association of Emergency Physicians. Position Statement on Emergency Department Overcrowding. CJEM.

[27] Powell, E.S., R.K. Khare, A.K. Venkatesh, B.D. Van Roo, J.G. Adams, G. Reinhardt. The relationship between inpatient discharge timing and emergency department boarding. The Journal of Emergency Medicine. 2012; 42: 186-196. PMid: 20888163. http: //dx.doi.org/10.1016/j.jemermed.2010.06.028

[28] Handel, D.A., A.A. Ginde, A.S. Raja, J. Rogers, A.F. Sullivan, J.A. Espinola, et al. Implementation of crowding solutions from the American College of Emergency Physicians Task Force Report on Boarding. International Journal of Emergency Medicine. 2010; 3: 279-286. PMid: 21373293. http://dx.doi.org/10.1007/s12 245-010-0216-6 